\title{
Demand bargaining and proportional payoffs in majority games*
}

\author{
Maria Montero $^{\dagger} \quad$ Juan J. Vidal-Puga ${ }^{\ddagger}$
}

May 18, 2010

\begin{abstract}
We study a majoritarian bargaining model in which players make payoff demands in decreasing order of voting weight. The unique subgame perfect equilibrium outcome is such that the minimal winning coalition of the players that move first forms with payoffs proportional to the voting weights. This result advances previous analysis in terms of one or more of the following: a) the simplicity of the extensive form (finite horizon with a predetermined order of moves); b) the range of the majority games covered; c) the equilibrium concept (subgame perfect equilibrium is sufficient for a unique prediction).
\end{abstract}

${ }^{*}$ We would like to thank Massimo Morelli, Alex Possajennikov, Daniel Seidmann, the editor, two referees, participants in the Midlands Game Theory Workshop and seminar participants at Queen's Belfast and Málaga for helpful comments. The second author acknowledges financial support from the Spanish Ministerio de Ciencia y Tecnología and FEDER through grant SEJ2005-07637-C02-01/ECON and from the Xunta de Galicia through grants PGIDIT06PXIC300184PN and PGIDT06PXIB362390.

${ }^{\dagger}$ Corresponding author. School of Economics, University of Nottingham, University Park, Nottingham NG7 2RD, United Kingdom. Phone + 44115 9515468, fax +44 115 951 4159, e-mail: maria.montero@nottingham.ac.uk.

${ }^{\ddagger}$ Research Group of Economic Analysis (RGEA) and Department of Statistics and Operations Research, University of Vigo. Facultad de Ciencias Sociales, Campus A Xunqueira, 36005 Pontevedra, Spain. Phone +34986 802014, fax +34986 813511, e-mail vidalpuga@uvigo.es. 
Key Words: Demand bargaining; coalition formation; weighted majority games; minimal winning coalitions.

J.E.L. Classification Numbers: C72, C78, D72. 


\section{Introduction}

Consider the classical problem of dividing a dollar by majority rule. Suppose there are $n$ parties, party $i$ has $\omega_{i}$ votes and $q$ votes $\left(q>\frac{\sum_{i \in N} \omega_{i}}{2}\right)$ are needed to achieve a majority. The relation between voting weights and actual payoff division is not straightforward. If there are three parties controlling 4, 3 and 2 votes respectively and 5 votes are needed to get a majority, the three parties are actually symmetric and one would expect a two-party coalition dividing the payoff equally. ${ }^{1}$ In other cases, ex post payoffs proportional to the number of votes seem natural. For example, let $\omega_{1}=2, \omega_{2}=\omega_{3}=$ $\omega_{4}=1$ and $q=3$. There are two kinds of minimal winning coalitions: the large party together with a small party, and all the small parties together (games with this property are called apex games). It is natural to assume that the three small parties would get $\frac{1}{3}$ each if they formed a coalition and that, since the large party can replace two small parties, it would receive their combined payoff, $\frac{2}{3}$, in the event of being in the coalition that forms.

Suppose the distribution of votes is such that the game is constant-sum (no ties are possible) and a homogeneous representation exists (even if not all minimal winning coalitions control the same number of votes, we can assign equivalent voting weights to the players so that all minimal winning coalitions have the same total voting weight). Assuming that a player and a group that can replace it in a minimal winning coalition must have the same payoff leads to a unique prediction for ex post payoffs: they must be proportional to the homogeneous voting weights (see Peleg [28], theorem 3.5, and Ostmann [27], lemma 4.9). Many cooperative solution concepts make this prediction, including von Neumann-Morgenstern's [33] main simple solution, the set of balanced aspirations (Cross [11]), the competitive solution (McKelvey et al. [20]) and the demand bargaining set (Morelli and Montero [24]).

\footnotetext{
${ }^{1}$ We assume that voters are not bound by fairness norms that would force them to divide the payoff proportionally to their number of votes; cf. Gamson [16].
} 
Despite proportional payoffs being intuitive and supported by many cooperative solution concepts, they are not supported by the leading noncooperative bargaining models. The most influential model of bargaining in majority games is the closed rule model of Baron and Ferejohn [3], which is based on Rubinstein [29] and Binmore [7]. ${ }^{2}$ In this model, a player is randomly selected to make a proposal specifying a payoff for each of the $n$ players. The model has a multiplicity of subgame perfect equilibria, and the one selected by refinements leads to a large proposer advantage. Hence, ex post payoffs are far from being proportional. Other proposal-based models lead to payoff divisions that tend to be egalitarian rather than proportional (Chatterjee et al. [10]) or predict that the first proposer gets everything (Bloch and Rottier [8]). ${ }^{3}$

An alternative approach to bargaining, introduced by Binmore [6] for three-player games and generalized by Selten [30], is based not on complete proposals but on demands. Players sequentially announce the payoff they require in order to participate in a coalition, and a coalition is formed by players making compatible demands. Because with demand bargaining a player is not always facing a unique proposal but may be able to compare different demands and choose the best coalition, one might expect the outcome of demand bargaining to be more competitive. Indeed, Binmore [6] calls his demand bargaining model a market bargaining model.

Morelli [23] uses a demand bargaining model to analyze weighted majority games. In this model, the Head of State chooses the first mover, and the latter chooses the order in which the parties formulate demands. This model was originally thought to yield proportional payoffs; however, extreme coun-

\footnotetext{
${ }^{2}$ This model has led to many applications and extensions. Recent related papers include Banks and Duggan [2], Diermeier et al. [13] and Kalandrakis [17].

${ }^{3}$ Proportionality results can be obtained in the context of the Baron-Ferejohn model (see Montero [21]). However, this proportionality is ex ante (ex post the proposer obtains more than half of the total payoff) and in order to hold generally it requires the recognition probabilities to be themselves proportional.
} 
terexamples were later found. Because the first mover chooses the order of moves, it may be able to play the remaining parties off against each other and obtain the whole payoff, even though the rules of the game allow the other parties to exclude the first mover (see Montero and Vidal-Puga [22]). ${ }^{4}$

In this paper we study a demand bargaining procedure in which the players must move in decreasing order of size. This procedure mirrors the assumption of Austen-Smith and Banks [1] that parties are asked to try to form a government in decreasing order of weight. Parties making demands in decreasing order of weight is not a completely unnatural assumption. In some countries the largest party must be asked to form the government first; in the absence of this rule the largest party is still selected quite often. ${ }^{5}$

We analyze situations in which the distribution of votes is well-behaved so that the resulting majority game is constant-sum and an equivalent homogeneous representation exists. We show that the subgame perfect equilibrium outcome is unique, and equilibrium payoffs inside the coalition that forms are proportional to the homogeneous voting weights.

The proportionality result is remarkable for its generality and for the simplicity of the bargaining procedure. Because players move in a fixed order, one might expect some players to find themselves in a favorable position in the sequence and exploit it (this is what happens in [22] and [8]). However, it turns out that moving by decreasing weight generates enough competitive pressure so that no player gets a disproportionate payoff within

\footnotetext{
${ }^{4}$ Other demand bargaining models in the literature would lead to multiple subgame perfect equilibria for majority games (Bennett and van Damme [5], Winter [34], Dasgupta and Chiu [12], and Vidal-Puga [32]) or have not been explored beyond symmetric games (Cardona-Coll and Mancera [9]) or apex games (Frechette et al. [15]).

${ }^{5}$ The Greek constitution prescribes that the leader of the largest party must be chosen as the first formateur; if he fails, the leader of the second largest party is selected, to be followed by the leader of the third largest party if he too fails. Even if the constitution is silent on this matter, a convention may emerge (Laver and Schofield, 1990, p. 210). Diermeier and Merlo (2004) analyze formateur selection empirically and find that the largest party is not necessarily the formateur but is disproportionally likely to be selected.
} 
the coalition that forms.

The proof is based on a recursive argument in which each player anticipates that a coalition will form that maximizes the benefit per vote of the remaining members in the coalition. This happens on and off the equilibrium path, and it happens despite substantial nonproportionality of demands off the equilibrium path.

The uniqueness of SPE payoffs result is far from trivial. Even though the game has a finite horizon, there are two potential sources of multiplicity. One is that the game is not finite since demands can be any real number between 0 and 1 . The other one is that players face choices between which they (but not other players!) are indifferent. ${ }^{6}$

If we change the order in which players move, the proportionality and uniqueness results break down. We also show that there are minimal winning coalitions that cannot form with a proportional division for any order of moves.

When the game is not constant-sum or an equivalent homogeneous representation does not exist, the reasons to expect proportional payoffs are weaker. The intuitive reasoning based on the substitutability of players in minimal winning coalitions may lead to several possible answers or to a contradiction, and the cooperative solution concepts do not necessarily predict proportionality. Neither does our demand bargaining model, as we show in section 4 .

\section{The model}

\subsection{Weighted majority games}

Consider a legislature in which $n$ parties are represented. We denote them as $N=\{1,2, \ldots, n\}$. There is a budget of size 1 to be divided by majority rule. Each player $i$ has $\omega_{i}$ votes, and a quota of $q$ is needed for a majority.

\footnotetext{
${ }^{6}$ The finite-horizon Baron-Ferejohn model does have multiple SPE (see Norman [26]).
} 
Denote $\left(\omega_{i}\right)_{i \in N}$ by $\omega$. The pair $[q ; \omega]$ is called a weighted majority game. Notice that the game is not affected if weights and quota are multiplied by the same positive constant.

Given a vector $x \in \mathbb{R}^{n}$ and a coalition $S \subset N$, we denote as $x_{S}$ the sum of the coordinates of the members of $S, x_{S}:=\sum_{i \in S} x_{i}$.

A coalition $S \subset N$ is winning if $\omega_{S} \geq q$; it is minimal winning if it is winning and no $T \varsubsetneqq S$ is winning. We denote as $W(q, \omega)$ the set of all winning coalitions, and as $W^{m}(q, \omega)$ the set of all minimal winning coalitions. We will omit the arguments $q$ and $\omega$ when no confusion can arise. A dummy player is one who does not belong to any minimal winning coalition.

A weighted majority game is constant-sum if $S \in W \Longleftrightarrow N \backslash S \notin W$ for all $S$. It admits an equivalent homogeneous representation if there exists a vector of votes $\omega^{h}$ and a quota $q^{h}$ such that $W^{m}(q, \omega)=W^{m}\left(q^{h}, \omega^{h}\right)=$ $\left\{S \subset N: \omega_{S}^{h}=q^{h}\right\}$. A weighted majority game that admits an equivalent homogeneous representation is called a homogeneous game.

Homogeneous representations are important because they better reflect the real bargaining power of the players. For example, in the game $[5 ; 4,3,2]$, any two players have a majority of votes, and the homogeneous representation $[2 ; 1,1,1]$ reflects this symmetry.

Homogeneous representations provide a "rate of exchange" between players that can be used as a basis for a competitive payoff division. If a homogeneous representation does not exist it is not obvious what a competitive payoff division would be. Consider the game $[5 ; 2,2,2,1,1,1]$, which has no homogeneous representation. A player with two votes can sometimes be replaced by a player with one vote (as in coalition $\{1,2,3\}$ ); in other cases two players with one vote are needed (as in coalition $\{1,2,4\}$ ).

Homogeneous representations are not necessarily unique. For example, $[5 ; 3,2,2,1]$ and $[7 ; 4,3,3,1]$ are two homogeneous representations of the same game. There are two kinds of minimal winning coalitions in this game: the large player with a medium-size player, and the three smaller 
players together. Homogeneity requires that the small player together with one of the medium-size players have the same weight as the large player; there is no restriction on the weight ratio between the medium-size and the small player as the medium-size player cannot be replaced by smaller players in a minimal winning coalition. Requiring that the game be constant-sum ensures that the set of minimal winning coalitions is sufficiently rich so that the homogeneous representation is unique whenever it exists. ${ }^{7}$

As will become clear from the analysis, dummy players must get 0 in equilibrium, so for simplicity we assume there are no dummy players. In what follows, we will take $[q ; \omega]$ to be the homogeneous representation with $\omega_{n}=1$; i.e. the weakest player has exactly 1 vote. Under these circumstances, every player in a constant-sum homogeneous game has a positive integer number of votes. Furthermore, $\omega_{N}=2 q-1$ and any $S$ that is maximal losing (i.e. $S$ is losing and no $T \nsupseteq S$ is losing) has $\omega_{S}=q-1$ (see Ostmann [27], lemma 4.11 and theorem 5.6).

\subsection{The bargaining procedure}

Let there be a constant-sum homogeneous game with homogeneous representation $[q ; \omega]$. There is a budget of size 1 to be divided. Player $i$ 's utility function is $u_{i}\left(x_{i}\right)$, where $x_{i}$ is $i$ 's share of the budget, and $u_{i}^{\prime}\left(x_{i}\right)>0$ for all $x_{i}$. Bargaining proceeds as follows. Players move in decreasing order of weight. We label the players in this order, so that player 1 moves first, followed by player 2 , etc. ${ }^{8}$

Each player $i$ makes a demand $d_{i}$, following the order of play, where $d_{i} \in[0,1]$ is the share of the budget player $i$ claims. If, after player $i$ makes

\footnotetext{
${ }^{7}$ This uniquess is up to multiplication by a positive constant and to the weight that is assigned to dummies, which may be 0 or a sufficiently small number (Peleg [28]).

${ }^{8}$ Of course, since it may be the case that $\omega_{i}=\omega_{j}$ for some $i$ and $j$, the bargaining procedure is uniquely defined only up to a permutation of players with the same weight. Notice that selection by decreasing seat share in legislative bargaining is a particular case of selection by decreasing voting weight.
} 
its demand, there exists a winning coalition $S \subset\{j: j \leq i\}$ such that $d_{S} \leq 1$, player $i$ has the option to form $S$, in which case payoffs are distributed according to the demands made. If there is more than one possible $S$, player $i$ decides which one is formed. ${ }^{9}$ If player $n$ forms no coalition, the game ends with each player getting zero. ${ }^{10}$ Thus, our bargaining procedure is identical to that in [12], except that the order of moves must be by decreasing weight rather than randomly determined at the start.

Given $i \in N$, we denote as $P_{i}$ the set of predecessors of $i$. Namely:

$$
P_{i}:=\{j \in N: j<i\} .
$$

If players move by decreasing weight, one can find a minimal winning coalition of players $\{1, \ldots, i\}$ that move consecutively, as the following lemma shows.

Lemma 1 Let $[q ; \omega]$ be a weighted majority game. There is a player $i$ such that $P_{i+1} \in W^{m}$.

Proof. Consider the smallest $i$ such that $S=\{1, \ldots, i\}$ is a winning coalition. If $S \notin W^{m}$, there must be a minimal winning coalition $S^{\prime} \subset S$, and $S^{\prime}$ is obtained from $S$ by deleting at least one player $j<i$. This is impossible because by assumption $\{1, \ldots, i-1\}$ is a losing coalition, and, since $\omega_{j} \geq \omega_{i}$ for all $j<i$, this coalition has at least as many votes as $S^{\prime}$.

We denote this minimal winning coalition of the players who move first by $S^{*}$. We now show that the total payoff is divided among the players in this coalition. We start by showing that any player who gets the move receives a positive payoff as long as there is still a positive payoff to be divided. Let $\mathbb{B}(d, i)$ with $d \in[0,1]^{i-1}$ be the subgame which begins when player $i$ has the move facing a vector of demands $d$. The complete game is $\mathbb{B}(\emptyset, 1)$.

\footnotetext{
${ }^{9}$ Note that player $i$ is not forced to form a coalition whenever feasible; this feature of the game is essential to ensure the existence of an SPE at all subgames (cf. example 1).

${ }^{10}$ Any finite number of bargaining rounds $T$ would lead to the same predictions.
} 
Proposition 1 Let $[q ; \omega]$ be a weighted majority game. Suppose we are in $\mathbb{B}(d, i)$ and $\min _{S \in W}\left\{d_{S \cap P_{i}}\right\}<1$. In any $S P E$ of this subgame a winning coalition $T \ni i$ is formed and $i$ receives at least

$$
m_{i}:=\frac{1-\min _{S \in W}\left\{d_{S \cap P_{i}}\right\}}{\left|N \backslash P_{i}\right|}>0 .
$$

Proof. We proceed by induction on $i$. For $i=n$, it is clear that $m_{i}$ is the payoff that player $n$ receives by forming a coalition $S \in W$ that minimizes $d_{S \cap P_{n}}$.

Assume the result holds for $i+1$. Suppose that player $i$ demands $m_{i}$. Then there is a positive payoff available to players moving after $i$, and under the induction hypothesis a winning coalition $S \ni i+1$ is formed and player $i+1$ must receive a positive amount. Let $j>i$ be the player that forms the winning coalition. If $i \in S$, the proof is done. If $i \notin S$, we have two cases:

If $j=i+1$, coalition $T=(S \backslash\{i+1\}) \cup\{i\}$ is also winning and player $i$ can form it, winning $1-d_{T \backslash\{i\}}$, which is clearly higher than $m_{i}$.

If $j>i+1$, consider the set $S \cap\{i+1, \ldots, j-1\}$ (which has at least one element, $i+1)$. Since player $j$ has chosen these players and not player $i$, and player $i$ has at least as many votes as any of them, we deduce that $d_{k} \leq m_{i}$ for all $k \in S \cap\{i+1, \ldots, j-1\}$. Let $l$ be the last of the players in $S \cap\{i+1, \ldots ., j-1\}$. Clearly, player $l$ receives $d_{l} \leq m_{i}$. However, this cannot be part of a SPE, because player $l$ could have formed the winning coalition $T=(S \backslash\{j\}) \cup\{i\}$, obtaining at least $2 m_{i}>d_{l}$.

Corollary 1 Players in $N \backslash S^{*}$ receive zero in any $S P E$ of $\mathbb{B}(\emptyset, 1)$.

Proof. It follows from proposition 1 that if a player $i \notin S^{*}$ receives a positive payoff, all its predecessors in $P_{i}$ must receive a positive payoff as well. But this cannot be part of a SPE, because the last player in $S^{*}$ would gain by deviating and forming $S^{*}$.

Corollary 2 If there are no veto players, all players in $S^{*}$ receive a positive payoff in any $S P E$ of $\mathbb{B}(\emptyset, 1)$. 
Proof. Proposition 1 implies that the set of players who get a positive payoff is a nonempty set of consecutive players starting from player 1 . Denote this set by $P_{i+1}$, where $i$ is the last player to receive a positive payoff. Because of the previous corollary, this set cannot be larger than $S^{*}$. Suppose it is smaller than $S^{*}$. Because there are no veto players, $N \backslash\{i\}$ is winning. Also, $d_{N \backslash\{i\} \cap P_{i+1}}<1$. Proposition 1 implies that $i+1$ gets a positive payoff, a contradiction.

If the game is constant-sum and homogeneous we are able to be more precise about equilibrium strategies and payoffs. Example 1 below illustrates the main features of equilibrium strategies for these games. Given the demands of the players that have moved so far, player $i$ determines two things: the optimal coalition to be (eventually) formed and the optimal demand to make. In general, the optimal coalition $S$ will control exactly $q$ votes, and will include some players that have moved before $i$, as well as some players moving after $i$. Since $T=S \cap P_{i}$ is a group of players that have already formulated a demand, $1-d_{T}$ is the benefit from buying the votes of the players in $T$; this benefit will be shared by the players in $S \backslash T$. Buying less votes leads to a higher benefit, but more votes from players moving after $i$ will be needed to complete a winning coalition. We will show that $S$ is chosen so that the average benefit per vote, $\frac{1-d_{T}}{q-\omega_{T}}$, is maximized.

The optimal demand for player $i$ will normally be $d_{i}=\omega_{i} \frac{1-d_{T}}{q-\omega_{T}}$, that is, player $i$ will claim a share of the benefit proportional to its number of votes. However, there are subgames off the equilibrium path in which player $i$ can demand more than a proportional share. Showing that player $i$ maximizes $\frac{1-d_{T}}{q-\omega_{T}}$ even though it does not always claim a proportional share of this benefit will be part of the challenge of the proof.

Example 1 also illustrates a feature of extensive form games with continuous action spaces: not every SPE of a subgame can be extended to an SPE of the overall game because ties cannot always be broken in an arbitrary way. Players may have to solve ties in a certain way in order to ensure that 
players moving earlier in the game have a best response (see [5]).

Example 1 Suppose there are five players, with 3,2,2,1 and 1 votes respectively, and the quota is 5 . Any SPE of $\mathbb{B}(\emptyset, 1)$ is such that coalition $\{1,2\}$ forms with $d_{1}=\frac{3}{5}$ and $d_{2}=\frac{2}{5}$.

Proof. At stage 5 , player 5 faces a vector of demands $\left(d_{1}, d_{2}, d_{3}, d_{4}\right)$. It has three choices:

a) Form coalition $\{1,4,5\}$ and get $1-d_{1}-d_{4}$.

b) Form coalition $\{2,3,5\}$ and get $1-d_{2}-d_{3}$.

c) Form no coalition and get $0 .^{11}$

Suppose one of the first two options is optimal. Then player 5 will form coalition $\{1,4,5\}$ if $1-d_{1}-d_{4} \geq 1-d_{2}-d_{3}$, or $d_{4} \leq d_{2}+d_{3}-d_{1}$. Ties are broken in favor of forming the coalition that includes player 4 , to guarantee that player 4 has a best response in the previous stage. Hence the maximum demand 4 can make and still get into a coalition with 5 is $d_{4}=d_{2}+d_{3}-d_{1}$.

At stage 4 , player 4 faces a vector of demands $\left(d_{1}, d_{2}, d_{3}\right)$. It can form coalition $\{2,3,4\}$ or make a demand that will lead to $\{1,4,5\}$. It forms $\{2,3,4\}$ if $1-d_{2}-d_{3} \geq d_{2}+d_{3}-d_{1}$, or equivalently

$$
1-d_{2}-d_{3} \geq \frac{1-d_{1}}{2} .
$$

Thus, player 4 is effectively comparing the average benefit associated to buying the votes of 2 and 3 (in which case 1 vote is enough to complete a winning coalition) or the votes of 1 (in which case 2 votes are needed to complete a winning coalition and 4 must share the benefit with 5 ).

From the inequality above, the maximum demand player 3 can make at the previous stage and still induce $\{2,3,4\}$ is

$$
d_{3}=\frac{1-2 d_{2}+d_{1}}{2} .
$$

\footnotetext{
${ }^{11}$ In order to simplify the proof, we only consider minimal winning coalitions. A coalition like $\{1,2,5\}$ could be relevant if $d_{2} \leq d_{4}$ and $1-d_{1}-d_{2}>0$, but this requires player 2 to have acted irrationally: player 2 would have been better-off by setting $d_{2}=1-d_{1}$ and forming coalition $\{1,2\}$.
} 
At stage 3 , player 3 faces a vector of demands $\left(d_{1}, d_{2}\right)$. It can form coalition $\{1,3\}$ or make a demand that will induce $\{2,3,4\}$. It makes a demand if $\frac{1-2 d_{2}+d_{1}}{2} \geq 1-d_{1}$, or equivalently

$$
\frac{1-d_{2}}{3} \geq \frac{1-d_{1}}{2}
$$

Again, player 3 may buy the votes of player 1 (in which case 2 votes are required to complete a winning coalition), or the votes of player 2 (in which case 3 votes are required to complete a winning coalition). It chooses the alternative with the highest average benefit.

The maximum demand player 2 can make in the previous stage and still induce coalition $\{2,3,4\}$ is

$$
d_{2}=\frac{3 d_{1}-1}{2}
$$

At stage 2, player 2 compares $1-d_{1}$ and $\frac{3 d_{1}-1}{2}$. It forms $\{1,2\}$ if $\frac{3 d_{1}-1}{2} \leq$ $1-d_{1}$, or $d_{1} \leq \frac{3}{5}$. This inequality can be rewritten as $\frac{1-d_{1}}{2} \geq \frac{1}{5}$ (where $\frac{1}{5}$ is the average benefit of buying no votes).

Anticipating this, player 1 sets $d_{1}=\frac{3}{5}$. Player 2 will then set $d_{2}=\frac{2}{5}$ and coalition $\{1,2\}$ is formed.

\section{$3 \quad$ The proportionality result}

In this section we present and prove the main result of the paper, namely that moving in decreasing order of weight leads to a competitive outcome (proportional payoffs within a minimal winning coalition) for well behaved games.

Theorem 1 Let $[q ; \omega]$ be a constant-sum homogeneous game. Suppose players play a demand bargaining game in decreasing order of weight. Then in any subgame perfect equilibrium coalition $S^{*}$ forms with each player $i$ demanding $\frac{\omega_{i}}{q}$. 
The result trivially follows if there is a veto player. In constant-sum games, a veto player must be a dictator, thus $\omega_{1}=q$, and player 1 can set $d_{1}=1$ and form coalition $\{1\}$. Assume from now on that $\omega_{i}<q$ for all $i$.

The proof of theorem 1 is divided into three parts: description of strategies that lead to coalition $S^{*}$ forming with proportional payoffs, showing that the strategies constitute an SPE of the game, and showing that equilibrium SPE payoffs are unique.

\subsection{Strategies}

We start by eliminating all strategies that involve strictly dominated choices of $d_{i}$. A demand $d_{i}$ is strictly dominated at $\mathbb{B}(d, i)$ if there exists a set $T \subset P_{i}$ such that $T \cup\{i\}$ is winning and $d_{i}<1-d_{T}$. By demanding less than it can get by forming a coalition, player $i$ gets a lower payoff regardless of the actions of players moving after $i$.

\section{Candidate coalitions}

Given the demands of the players that have moved so far, player $i$ determines two things: the optimal winning coalition to be (eventually) formed and the optimal demand to make. Player $i$ will consider winning coalitions $S$ that include some players from $N \backslash P_{i}$ (player $i$ and possibly players that move after it) and may also include some predecessors from $P_{i}$. Let $\alpha$ be the number of votes controlled by players in $S \cap\left(N \backslash P_{i}\right)$. The players in $S \cap P_{i}$ must control at least $q-\alpha$ votes. We denote by $b(i, \alpha)$ the maximum benefit that can be achieved by buying these $q-\alpha$ votes from players in $P_{i}$.

$$
b(i, \alpha):=\max \left\{1-d_{T}: T \subset P_{i}, \omega_{T} \geq q-\alpha\right\} .
$$

Player $i$ can calculate $b(i, \alpha)$ for every feasible value of $\alpha$. Not all integers between 0 and $q$ are feasible for every player: $\alpha$ cannot be so small that even the votes of all the players in $P_{i}$ would not suffice, and it cannot exceed the votes of all players in $N \backslash P_{i}$. Let $\gamma_{0}^{i}:=\max \left(q-\omega_{P_{i}}, 0\right)$ and $\delta^{i}:=\omega_{N \backslash P_{i}}$. In order for $b(i, \alpha)$ to exist we need $\gamma_{0}^{i} \leq \alpha \leq \delta^{i}$. Player $i$ will consider values 
of $\alpha$ between $\gamma^{i}=\max \left\{1, \gamma_{0}^{i}\right\}$ and $\delta^{i}$. The lower bound of 1 appears because player $i$ only considers coalitions with $S \ni i$, and $\omega_{i} \geq 1$.

Notice that $\delta^{i+1}=\delta^{i}-\omega_{i}$ for all $i<n$. Also, $\omega_{i}<q$ implies $\gamma_{0}^{i} \leq \delta^{i+1}$. It follows from lemma 4.9 in Ostmann [27] that $\omega_{i} \leq \delta^{i+1}$ for all $i<n$, thus $\gamma^{i} \leq \delta^{i+1}$ for all $i<n$.

For player 1 only $\alpha \geq q$ is feasible and $b(1, \alpha)=1$ for all $\alpha \geq q$. For player $n$, only $\alpha=1$ is feasible and $b(n, 1)$ is simply $n$ 's payoff from buying the votes of one of the cheapest coalitions controlling at least $q-1$ votes.

We denote as $\Sigma^{i}$ the set of values between $\gamma^{i}$ and $\delta^{i}$ that maximize $b(i, \alpha) / \alpha$. A generic element of $\Sigma^{i}$ is denoted by $\sigma^{i}$. Thus:

$$
\Sigma^{i}:=\underset{\gamma^{i} \leq \alpha \leq \delta^{i}}{\arg \max } \frac{b(i, \alpha)}{\alpha} .
$$

Obviously, the set $\Sigma^{i}$ depends on the demands made by the players in $P_{i}$. For example, in the game $[4 ; 3,1,1,1,1], d_{1}=\frac{1}{2}$ leads to $\Sigma^{2}=\{1\}$, and $d_{1}=\frac{3}{4}$ leads to $\Sigma^{2}=\{1,4\}$.

The following lemma will be central to the proof. It shows that all values of $\alpha$ between $\delta^{i+1}+1$ and $\delta^{i+1}+\omega_{i}=\delta^{i}$ lead to the same $b(i, \alpha)$. The extra votes are not valuable because they are not enough to replace any player from $P_{i}$. For example, in the game $[10 ; 7,3,3,3,1,1,1], \delta^{4}=6$ and $\delta^{5}=3$. Consider the situation of player 4 . If it takes $\alpha=4$, there are two ways to form a winning coalition: buying the votes of player 1 , or buying the votes of players 2 and 3 . Thus, $b(4,4)=\max \left(1-d_{1}, 1-d_{2}-d_{3}\right)$. If instead it takes $\alpha=5$ or $\alpha=6$, exactly the same players are needed: none of the predecessors of player 4 can be dispensed with despite the extra votes. Note that the assumptions of homogeneity and the game being constant-sum are used in the proof of this lemma.

Lemma 2 Suppose we are in the subgame $\mathbb{B}(d, i)$. Then

$$
\left\{T \subset P_{i}: \omega_{T} \geq q-\left(\delta^{i+1}+\beta\right)\right\}=\left\{T \subset P_{i}: \omega_{T} \geq q-\delta^{i}\right\}
$$

for all $\beta=1,2, \ldots, \omega_{i}$. 
Proof. " $\subset$ " is left to the reader.

" $\supset$ " Let $T \subset P_{i}$ such that $\omega_{T} \geq q-\delta^{i}$. Then, $T \cup\left(N \backslash P_{i}\right)$ is winning and contains player $i$. There are two possibilities:

If $T \cup\left(N \backslash P_{i+1}\right)$ is also winning, $\omega_{T} \geq q-\delta^{i+1}$ and the result follows.

If $T \cup\left(N \backslash P_{i+1}\right)$ is losing, its complement, $(N \backslash T) \cap P_{i+1}$, is winning and contains player $i$ as the weakest member. By taking out player $i$, we obtain the coalition $(N \backslash T) \cap P_{i}$ which is losing (since its complement $T \cup\left(N \backslash P_{i}\right)$ is winning). Thus, $(N \backslash T) \cap P_{i+1}$ is minimal winning and $T \cup\left(N \backslash P_{i+1}\right)$ is maximal losing. Because maximal losing coalitions control $q-1$ votes, $\omega_{T}=q-\omega_{N \backslash P_{i+1}}-1 \geq q-\omega_{N \backslash P_{i+1}}-\beta$.

Corollary 3 In $\mathbb{B}(d, i)$, we have $b\left(i, \delta^{i+1}+\beta\right)=b\left(i, \delta^{i}\right)$ for all $\beta=1,2, \ldots, \omega_{i}$. Moreover, for all $\sigma^{i} \in \Sigma^{i}$, if $b\left(i, \sigma^{i}\right)>0$,

$$
\sigma^{i}>\delta^{i+1} \Longrightarrow \sigma^{i}=\delta^{i+1}+1
$$

There are three possible cases for $b\left(i, \sigma^{i}\right)$, depending on whether it is greater, equal or less than 0 .

If $b\left(i, \sigma^{i}\right)<0$, it is no longer possible to form a winning coalition and any $d_{i} \geq 0$ would be optimal for player $i$. We will set $d_{i}=0$. An example of this type of subgame occurs for $[5 ; 3,2,2,1,1]$ if $d_{1}=d_{2}=d_{3}=d_{4}=\frac{3}{4}$.

If $b\left(i, \sigma^{i}\right) \geq 0$, a coalition can still be formed. ${ }^{12}$ Player $i$ will use the set $\Sigma^{i}$ to determine which coalition will be formed. The set $\Sigma^{i}$ will not generally produce a unique answer but a set of candidate coalitions, one of which will eventually be formed. Intuitively, a candidate coalition should be of the form $S \cup\{i, \ldots, i+l\}$, where $S \in \arg \max _{T \subset P_{i}: \omega_{T} \geq q-\sigma^{i}} 1-d_{T}$ is the group of players that have already moved whose votes will be bought, $\{i, \ldots, i+l\}$ is a set of consecutive players that control exactly $\sigma^{i}$ votes, and $\sigma^{i} \in \Sigma^{i}$. The reason why $\{i, \ldots, i+l\}$ ought to be a group of consecutive players is that

\footnotetext{
${ }^{12}$ If $b\left(i, \sigma^{i}\right)=0$, there is nothing at stake for player $i$ but we still need to specify $i$ 's strategy carefully in order to ensure that previous movers have a best response.
} 
any plans to exclude a player between $i$ and $i+l$ may well be sabotaged by this player when it gets the move.

It is not immediate that coalitions satisfying the above conditions always exist. For example, in the game $[7 ; 4,3,2,1,1,1,1]$, finding $\Sigma^{2}=\{1\}$ or $\Sigma^{2}=\{4\}$ would preclude their existence, and $\Sigma^{2}$ would not be a reliable guide to choosing a coalition. We now show that a coalition can always be constructed as described above. We start by showing that, given homogeneity and our elimination of strictly dominated demands, there can be no positive benefit from setting $\alpha<\omega_{i}$.

Lemma 3 Let $\gamma_{0}^{i} \leq \alpha<\omega_{i}$. Then $b(i, \alpha) \leq 0$. Moreover, $b(i, \alpha)=0$ implies $b\left(i, \omega_{i}\right) \geq 0$.

Proof. Let $T \subset P_{i}$ such that $\omega_{T} \geq q-\alpha$. Since $\alpha<\omega_{i}$, we have

$$
\omega_{T \cup\{i\}}=\omega_{T}+\omega_{i}>\omega_{T}+\alpha \geq q .
$$

Hence, since the game is homogeneous, $T \cup\{i\}$ cannot be minimal winning. Moreover, player $i$ is the player with the least votes in $T \cup\{i\}$, thus coalition $T$ should be winning. This means that either $d_{T} \geq 1$ (implying $b(i, \alpha) \leq 0)$ or $d_{T}<1$, in which case the smallest player in $T$ (player $j$ ) would have been strictly better-off by setting a higher demand and forming a coalition, regardless of the actions of the players moving after $j$. The latter case is ruled out by our elimination of strictly dominated demands.

When $b(i, \alpha)=0, b\left(i, \omega_{i}\right) \geq 0$ follows from the fact that $b(i, \cdot)$ is nondecreasing in the second variable.

The case $b\left(i, \sigma^{i}\right)>0$ is the most important one since there is a positive benefit available to the players in $N \backslash P_{i}$. In this case, all elements of $\Sigma^{i}$ are associated to at least one candidate coalition, and all such coalitions are minimal winning as the following corollary shows.

Corollary 4 Suppose $b\left(i, \sigma^{i}\right)>0$ for some $/$ all $\sigma^{i} \in \Sigma^{i}$. Then for all $\sigma^{i} \in \Sigma^{i}$ a) $\sigma^{i} \geq \omega_{i}$. 
b) There is a set of consecutive players $\{i, \ldots, i+l\}$ with exactly $\sigma^{i}$ votes.

c) For all $S \in \arg \max _{T \subset P_{i}: \omega_{T} \geq q-\sigma^{i}} 1-d_{T}$, it holds that $\sigma^{i}+\omega_{S}=q$.

Proof. Part a) follows since $b(i, \alpha) \leq 0$ for all $\alpha<\omega_{i}$. Part c) follows because if $\sigma^{i}+\omega_{S}>q, \sigma^{i}-1$ would lead to a higher benefit per vote and $\sigma^{i} \notin \Sigma^{i}$. Given that $\sigma^{i}+\omega_{S}=q$, b) follows from homogeneity.

For example, with votes $[3 ; 2,1,1,1]$ and $d_{1}=\frac{2}{3}, \Sigma^{2}=\{1,3\}$ and the candidate coalitions are $\{1,2\}$ and $\{2,3,4\}$. With votes $[4 ; 2,2,1,1,1]$ and $d_{1}=d_{2}=\frac{2}{3}, \Sigma^{3}=\{2\}$ and the candidate coalitions are $\{1,3,4\}$ and $\{2,3,4\}$.

For $b\left(i, \sigma^{i}\right)=0$, candidate coalitions can always be constructed since $\delta^{i} \in \Sigma^{i}$ and $\{i, \ldots, i+n\}$ control exactly $\delta^{i}$ votes. The difference with the case $b\left(i, \sigma^{i}\right)>0$ is that some $\sigma^{i} \in \Sigma^{i}$ may not be associated to a coalition, and candidate coalitions may not be minimal winning. This is of no consequence since there is nothing at stake for player $i$ or its successors.

\section{Formulating a demand}

If $b\left(i, \sigma^{i}\right)<0$, player $i$ sets $d_{i}=0$. Suppose henceforth that $b\left(i, \sigma^{i}\right) \geq 0$.

Player $i$ expects that one of the candidate coalitions will be formed, and formulates a demand accordingly. Player $i$ demands at least $\omega_{i} \frac{b\left(i, \sigma^{i}\right)}{\sigma^{i}}$ (a share of the benefit proportional to $i$ 's votes), but may demand more depending on what player $i+1$ can achieve without player $i$. If player $i+1$ decides to exclude player $i$, it is in a similar situation to player $i$ but with less feasible values for $\alpha$. It would choose an $\alpha$ between $\gamma^{i}$ and $\delta^{i+1}$, and the maximum benefit from buying $q-\alpha$ votes without buying the votes of player $i$ is precisely $b(i, \alpha)$. We define $T^{i}$ as the set of values between $\gamma^{i}$ and $\delta^{i+1}$ that maximize $\frac{b(i, \alpha)}{\alpha}$ (recall that $\gamma^{i} \leq \delta^{i+1}$, so the interval is nonempty).

$$
T^{i}:=\underset{\gamma^{i} \leq \alpha \leq \delta^{i+1}}{\arg \max } \frac{b(i, \alpha)}{\alpha} .
$$

Let $\tau^{i} \in T^{i}$. Because $\gamma^{i} \leq \tau^{i} \leq \delta^{i+1}<\delta^{i}, \frac{b\left(i, \tau^{i}\right)}{\tau^{i}} \leq \frac{b\left(i, \sigma^{i}\right)}{\sigma^{i}}$ for all $\sigma^{i} \in \Sigma^{i}$. 
For any values of $\sigma^{i} \in \Sigma^{i}$ and $\tau^{i} \in T^{i}$, we define

$$
d_{i}^{*}:=\left\{\begin{array}{ll}
\frac{\omega_{i} b\left(i, \sigma^{i}\right)}{\sigma^{i}} & \text { if } \sigma^{i} \leq \delta^{i+1} \\
b\left(i, \sigma^{i}\right)-\frac{\sigma^{i}-\omega_{i}}{\tau^{i}} b\left(i, \tau^{i}\right) & \text { if } \sigma^{i}>\delta^{i+1} \text { and } b\left(i, \tau^{i}\right) \geq 0 \\
b\left(i, \sigma^{i}\right) & \text { if } \sigma^{i}>\delta^{i+1} \text { and } b\left(i, \tau^{i}\right)<0 .
\end{array}\right\}
$$

Intuitively, player $i$ wants to form a coalition with $\alpha=\sigma^{i}$ and to claim as much of $b\left(i, \sigma^{i}\right)$ as possible, but it must take into account that player $i+1$ can obtain a benefit per vote of $\frac{b\left(i, \tau^{i}\right)}{\tau^{i}}$ without player $i$. If $b\left(i, \tau^{i}\right)<0$, player $i+1$ is helpless and player $i$ can claim the whole $b\left(i, \sigma^{i}\right)$. Otherwise, player $i$ must allow a benefit of $\frac{b\left(i, \tau^{i}\right)}{\tau^{i}} \leq \frac{b\left(i, \sigma^{i}\right)}{\sigma^{i}}$ per vote for the players moving after it that will provide the remaining $\sigma^{i}-\omega_{i}$ votes, thus $d_{i}=$ $b\left(i, \sigma^{i}\right)-\frac{\sigma^{i}-\omega_{i}}{\tau^{i}} b\left(i, \tau^{i}\right)$. Furthermore, if $\sigma^{i} \leq \delta^{i+1}$, player $i+1$ can obtain a benefit of $\frac{b\left(i, \sigma^{i}\right)}{\sigma^{i}}$ per vote without resorting to buying the votes of player $i$ and thus $\frac{b\left(i, \tau^{i}\right)}{\tau^{i}}=\frac{b\left(i, \sigma^{i}\right)}{\sigma^{i}}$ and $b\left(i, \sigma^{i}\right)-\frac{\sigma^{i}-\omega_{i}}{\tau^{i}} b\left(i, \tau^{i}\right)=\frac{\omega_{i} b\left(i, \sigma^{i}\right)}{\sigma^{i}}$.

Note that $d_{i}^{*}$ is defined irrespective of whether player $i$ forms a coalition after formulating this demand. Also, $d_{i}^{*}$ is independent of the particular choice of $\sigma^{i} \in \Sigma^{i}$ and $\tau^{i} \in T^{i}$. By definition, $\frac{b\left(i, \tau^{i}\right)}{\tau^{i}}$ and $\frac{b\left(i, \sigma^{i}\right)}{\sigma^{i}}$ are independent of the $\tau^{i}$ and $\sigma^{i}$ chosen. Also, $b\left(i, \tau^{i}\right) \geq 0$ for some $\tau^{i} \in T^{i}$ if and only if $b\left(i, \tau^{i}\right) \geq 0$ for all $\tau^{i} \in T^{i}$. If $b\left(i, \sigma^{i}\right)=0$ for some $\sigma^{i}$, then $b\left(i, \sigma^{i}\right)=0$ for all $\sigma^{i}$ and $b\left(i, \tau^{i}\right) \leq 0$ for all $\tau^{i} \in T^{i}$. Thus, $d_{i}^{*}=0$ regardless of the choice of $\sigma^{i}$ and $\tau^{i}$. If $b\left(i, \sigma^{i}\right)>0, d_{i}^{*}$ is the same for all $\sigma^{i} \leq \delta^{i+1}$. If $\sigma^{i}>\delta^{i+1}, \sigma^{i}=\delta^{i+1}+1$. If $\Sigma^{i}$ contains some $\sigma^{i} \leq \delta^{i+1}$ as well as $\sigma^{i}>\delta^{i+1}$, $d_{i}^{*}$ is still independent of the choice of $\sigma^{i}$ because $\frac{b\left(i, \tau^{i}\right)}{\tau^{i}}=\frac{b\left(i, \sigma^{i}\right)}{\sigma^{i}}$ in this case.

Note that player $i$ chooses a coalition based on maximizing $\frac{b\left(i, \sigma^{i}\right)}{\sigma^{i}}$, even though players moving after $i$ are paid at a potentially lower rate. Shouldn't player $i$ take more votes from $N \backslash P_{i+1}$ given that it needs to pay these players less? The answer is no. The inequality $\frac{b\left(i, \tau^{i}\right)}{\tau^{i}}<\frac{b\left(i, \sigma^{i}\right)}{\sigma^{i}}$ is only possible when $\sigma^{i}>\delta^{i+1}$ for all $\sigma^{i} \in \Sigma^{i}$. Then $\sigma^{i}=\delta^{i+1}+1$ and, because of lemma 2 , additional votes from $N \backslash P_{i}$ are of no use at all. 
To illustrate the demands made at different subgames, consider the weighted majority game $[5 ; 3,2,2,1,1]$. If $d_{1}=\frac{8}{10}$ and $d_{2}=\frac{7}{10}$, the maximum benefit per vote player 3 can achieve is $\frac{1}{10}$. There are two values of $\alpha$ that achieve this benefit per vote: $\alpha=2$ (with coalition $\{1,3\}$ in mind) and $\alpha=3$ (with coalition $\{2,3,4\}$ in mind). Player 3's demand depends on what player 4 can do without player 3 . Without player 3 , player 4 can still set $\alpha=2$ (with coalition $\{1,4,5\}$ in mind) and get $\frac{1}{10}$, thus $\frac{b\left(3, \tau^{3}\right)}{\tau^{3}}=\frac{1}{10}$ and $d_{3}=\omega_{3} \frac{b\left(3, \sigma^{3}\right)}{\sigma^{3}}$, which in this case is $\frac{2}{10}$. Alternatively, suppose $d_{1}=\frac{9}{10}$ and $d_{2}=\frac{7}{10}$. Now $\sigma^{3}=3$ is the only optimal value for $\alpha$, leading to a benefit per vote of $\frac{1}{10}$. The best player 4 can do without player 3 is still to set $\alpha=2$ with coalition $\{1,4,5\}$ in mind, but the benefit per vote is only $\frac{1-\frac{9}{10}}{2}=\frac{1}{20}$. Player 3 sets $d_{3}=\frac{3}{10}-\frac{1}{20}=\frac{1}{4}$ in this case.

If $d_{1}=d_{2}=d_{3}=\frac{3}{4}$, player 4 finds $\sigma^{4}=2$, corresponding to a total benefit of $\frac{1}{4}$ from buying the votes of player 1 , and a benefit per vote of $\frac{1}{8}$. Player 5 cannot do anything without player 4 (it would need to buy the votes of players whose demands add up to more than 1$)$, thus $b\left(4, \tau^{4}\right)<0$ and player 4 does not allow for any payoff for player 5 and sets $d_{4}=\frac{1}{4}$.

\section{Tie-breaking rule}

In order to complete the description of the strategies, it remains to specify which of the coalitions associated to $\Sigma^{i}$ is eventually formed.

Players will use the following tie-breaking rule: Given a player $i$ and a set of coalitions $\mathbb{S}$, player $i$ selects an element of $\mathbb{S}$ in the following way: First, select only the coalitions that contain the player with the highest index preceding $i$ (player $i-1$, or, if player $i-1$ is in none of the coalitions, player $i-2$ etc.). If there are several coalitions containing this player, select the ones that contain the player with the second highest index, etc. The tie-breaking rule will ensure that players moving before $i$ always have a best response (cf. example 1).

\section{Inductive definition of strategies}

In $\mathbb{B}(d, n)$, player $n$ sets $d_{n}=0$ and forms no coalition if $b(n, 1)<0$. 
Otherwise, player $n$ forms a coalition $S \cup\{n\}$ with $S \in \underset{T \subset P_{n}: \omega_{T} \geq q-\omega_{n}}{\arg \max }\left(1-d_{T}\right)$ after demanding $d_{n}=1-d_{S}$. If there is more than one possible choice of $S$, player $n$ uses the tie-breaking rule.

Let $i<n$ and assume we have defined the strategies for players in $\mathbb{B}(d, i+1)$. In $\mathbb{B}(d, i)$, player $i$ sets $d_{i}=0$ and forms no coalition if $b\left(i, \sigma^{i}\right)<$ 0 . Otherwise, player $i$ proceeds as follows:

1. If $\sigma^{i}>\omega_{i}$ for all $\sigma^{i} \in \Sigma^{i}$, player $i$ demands $d_{i}=d_{i}^{*}$ given as in (1).

2. If $\Sigma^{i}=\left\{\omega_{i}\right\}$, player $i$ forms coalition $S \cup\{i\}$ with $S \in \underset{T \subset P_{i}: \omega_{T} \geq q-\omega_{i}}{\arg \min } d_{T}$. If there is more than one possible choice of $S$, player $i$ uses the tiebreaking rule.

3. If $\left\{\omega_{i}\right\} \varsubsetneqq \Sigma^{i}$, player $i$ can anticipate the coalition $S^{\prime}$ that would be formed should it demand $d_{i}^{*}$ and form no coalition, given that its followers play the strategies we have defined.

(a) If $i \notin S^{\prime}$, player $i$ forms coalition $S \cup\{i\}$ with $S \in \underset{T \subset P_{i}: \omega_{T}>q-\omega_{i}}{\arg \min } d_{T}$. If there is more than one possible $S$, player $i$ uses the tie-breaking rule.

(b) If $i \in S^{\prime}$, player $i$ compares the coalitions $S \in \underset{T \subset P_{i}: \omega_{T} \geq q-\omega_{i}}{\arg \max }\left(1-d_{T}\right)$ and $S^{\prime} \cap P_{i}$. Among them, player $i$ selects a coalition following the tie-breaking rule. If $S^{\prime}$ is chosen, player $i$ demands $d_{i}=d_{i}^{*}$ given as in (1). If $S \neq S^{\prime}$ is chosen, then player $i$ demands $1-d_{S}=b\left(i, \omega_{i}\right)=d_{i}^{*}$ and forms coalition $S \cup\{i\} .{ }^{13}$

These strategies lead to proportional payoffs as the following lemma shows.

Lemma 4 If players follow the strategies described above, coalition $S^{*}$ forms with each player demanding $d_{i}=\frac{\omega_{i}}{q}$.

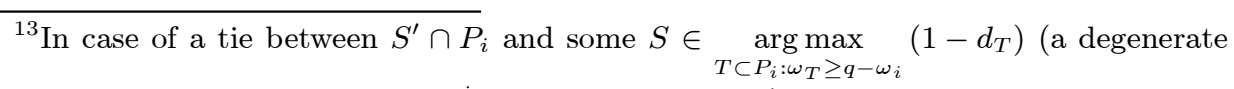
case that can only happen if $\left.b\left(i, \sigma^{i}\right)=0\right) S$ is chosen $\left(S^{\prime}\right.$ would do just as well).
} 
Proof. Because of lemma $1, S^{*}=P_{l+1}$ for some value of $l$. We can show $d_{i}=\frac{\omega_{i}}{q}$ for $i=1, \ldots, l$ by induction on $i$.

Player 1 finds $\Sigma^{1}=\{q\}$ and, since $q \leq \delta^{2}$ (due to the absence of veto players) sets a demand $d_{1}^{*}=\frac{\omega_{1}}{q}$. Given this demand, $q-\omega_{1} \in \Sigma^{2}$.

Assume now $d_{j}=\frac{\omega_{j}}{q}$ for all $j \in P_{i}$, and $q-\omega_{P_{i}} \in \Sigma^{i}$. Then, since $q-\omega_{P_{i}} \leq \delta^{i+1}$,

$$
d_{i}^{*}=\frac{\omega_{i} b\left(i, q-\omega_{P_{i}}\right)}{q-\omega_{P_{i}}}=\frac{\omega_{i}\left(1-\frac{\omega_{P_{i}}}{q}\right)}{q-\omega_{P_{i}}}=\frac{\omega_{i}}{q} .
$$

If $i<l, q-\omega_{P_{i+1}} \in \Sigma^{i+1}$ and induction continues. If $i=l, S^{*}$ is formed because of the tie-breaking rule.

We now illustrate how the strategies would work off the equilibrium path with votes $[5 ; 3,2,2,1,1]$. Let $d_{1}=\frac{8}{10}>\frac{\omega_{1}}{q}$.

Facing $d_{1}=\frac{8}{10}$, player 2 can form $\{1,2\}$ and obtain a benefit per vote of $\frac{1}{10}$. Excluding player 1 and setting $\alpha=5$ leads to a benefit per vote of $\frac{1}{5}$. Since $5=\sigma^{2}>\omega_{2}$ (case 1), player 2 does not form a coalition. Player 2's demand depends on what player 3 can do without player 2 . Without player 2 , player 3 is not able to achieve a benefit per vote of $\frac{1}{5}\left(5=\sigma^{2}>\delta^{3}=4\right)$. The best player 3 can do is set $\alpha=2$, with a benefit per vote of $\frac{1}{10}$. Player 2 allows a payoff of $\frac{1}{10}$ for each of the three votes it needs to complete the winning coalition, and sets $d_{2}=1-\frac{3}{10}=\frac{7}{10}$.

Facing $d_{1}=\frac{8}{10}$ and $d_{2}=\frac{7}{10}$, player 3 finds $\Sigma^{3}=\{2,3\}$ and $d_{3}=\frac{2}{10}$ (as discussed on p. 20). We are in case 3 since $\omega_{3} \varsubsetneqq \Sigma^{3}$. Player 3 compares the coalitions that can be formed immediately $(\{1,3\}$ in this case) and the coalition that would result otherwise. To determine the latter, we need to solve the subgame that arises if $d_{3}=\frac{2}{10}$ and no coalition is formed.

Suppose no coalition is formed, so that player 4 faces $d_{1}=\frac{8}{10}, d_{2}=\frac{7}{10}$ and $d_{3}=\frac{2}{10}$. The maximum benefit per vote that can be achieved is $\frac{1}{10}$, and $\Sigma^{4}=\{1,2\}$. We are in case 3 since $\omega_{4} \varsubsetneqq \Sigma^{4}$. Because player 5 can still achieve a benefit of $\frac{1}{10}$ without player 4 in coalition $\{2,3,5\}, d_{4}=\frac{1}{10}$. Player 4 compares forming coalition $\{2,3,4\}$ and forming no coalition. Again we 
need to solve the subgame that arises if $d_{4}=\frac{1}{10}$ and no coalition is formed.

Suppose player 5 faces $d_{1}=\frac{8}{10}, d_{2}=\frac{7}{10}, d_{3}=\frac{2}{10}$ and $d_{4}=\frac{1}{10}$. It is trivially in the case $\Sigma^{5}=\omega_{5}$, with a maximum benefit per vote of $\frac{1}{10}$ which can be achieved by two coalitions: $\{1,4,5\}$ and $\{2,3,5\}$. The tie-breaking rule indicates that $\{1,4,5\}$ is formed, since it includes player 4 .

Now we can apply the tie-breaking rule in earlier subgames to see what coalition is formed. Anticipating that player 5 would form $\{1,4,5\}$, player 4 forms $\{2,3,4\}$ because it contains player 3 . Anticipating this, player 3 compares $\{1,3\}$ with $\{2,3,4\}$ and does not form a coalition since $\{2,3,4\}$ contains 2. Thus, after player 1 demands $\frac{8}{10}$, coalition $\{2,3,4\}$ forms with payoff division $\left(\frac{7}{10}, \frac{2}{10}, \frac{1}{10}\right)$. Note that proportionality is not respected off the equilibrium path in this example.

\subsection{Showing that the described strategies constitute an SPE}

Consider any subgame $\mathbb{B}(d, i)$. We start by noting that, if $b\left(i, \sigma^{i}\right)<0$, all players get 0 in any SPE of the game. Hence, $d_{i}=0$ is trivially optimal.

Lemma 5 If $b\left(i, \sigma^{i}\right)<0$ for some/all $\sigma^{i} \in \Sigma^{i}$, any $d_{i} \geq 0$ is optimal and any Nash equilibrium of $\mathbb{B}(d, i)$ is such that all players get 0 .

Proof. Since $b\left(i, \sigma^{i}\right) / \sigma^{i}$ is maximum, we deduce that $b(i, \alpha)<0$ for every $\alpha \geq \gamma^{i}$. The same occurs for $\alpha=0$ since $b(i, \alpha)$ is nondecreasing in $\alpha$. This means that no winning coalition can be formed.

Suppose $b\left(i, \sigma^{i}\right) \geq 0$ for the rest of this section. We need to prove that, given that the remaining players follow the strategies described, player $i$ 's equilibrium demand is $d_{i}^{*}$. There are two parts to this result, both of which will be proven by induction over $i$ in proposition 2 :

1. Player $i$ always gets $d_{i}^{*}$ if it follows the prescribed strategy. This is obvious if player $i$ 's strategy requires player $i$ to form a coalition. If the strategy requires that $i$ does not form a coalition (which can only 
happen if $\sigma^{i}>\omega_{i}$ for some $\sigma^{i} \in \Sigma^{i}$ ), player $i$ is relying on other players to include it in a coalition. There are some auxiliary lemmas that will help us to prove by induction that $i$ gets $d_{i}^{*}$ in this case. The lemmas essentially state that player $i+1$ 's plans do not contradict $i$ 's plans: $\Sigma^{i+1}$ contains $\sigma^{i}-\omega_{i}$, and candidate coalitions for $i$ and $i+1$ are compatible. This is proven in the following steps:

(a) Lemma 6 shows how $b(i+1, \alpha)$ is determined from $b(i, \cdot)$ and $d_{i}$.

(b) Lemmas 7 and 8 together establish that $i+1$ maximizes benefit per vote by setting $\alpha=\sigma^{i}-\omega_{i}$ and buying the votes of player $i$.

(c) Lemma 9 shows that, if we limit ourselves to candidate coalitions involving $i$ and $i+1$, no coalitions are gained or lost when the turn moves from $i$ to $i+1$.

2. Player $i$ would get 0 if it deviates by setting $d_{i}>d_{i}^{*}$. Lemma 10 provides the base for induction by establishing that $i$ is not in any of $i+1$ 's candidate coalitions.

Consider the calculation of $b(i+1, \alpha)$ for an arbitrary $\alpha$. It may be the case that, having $\alpha$ votes in its pocket, player $i+1$ cannot form a winning coalition without player $i$. Then $b(i+1, \alpha)=b\left(i, \alpha+\omega_{i}\right)-d_{i}$ irrespective of $d_{i}$. Otherwise player $i+1$ will compare the best coalition that includes $i$ with the best coalition that does not include $i$. Given that $i$ is included in the coalition, $i+1$ needs to buy the remaining $q-\left(\alpha+\omega_{i}\right)$ votes from $P_{i}$, and the best way to do this leads to a benefit of $b\left(i, \alpha+\omega_{i}\right)$; after paying $d_{i}$, there is $b\left(i, \alpha+\omega_{i}\right)-d_{i}$ left. Without player $i$, the maximum benefit from buying $q-\alpha$ votes without buying $i$ 's votes is precisely $b(i, \alpha)$. Lemma 6 summarizes this result.

Lemma 6 Suppose we are in $\mathbb{B}(d, i+1)$. Let $\alpha$ such that $\gamma_{0}^{i+1} \leq \alpha \leq \delta^{i+1}$. Then $\gamma_{0}^{i} \leq \alpha+\omega_{i} \leq \delta^{i}$ and furthermore

a) if $\alpha<\gamma_{0}^{i}$, then $b(i+1, \alpha)=b\left(i, \alpha+\omega_{i}\right)-d_{i}$; 
b) if $\alpha \geq \gamma_{0}^{i}$, then $b(i, \alpha)$ exists and

$$
b(i+1, \alpha)=\max \left\{b(i, \alpha), b\left(i, \alpha+\omega_{i}\right)-d_{i}\right\} .
$$

\section{Proof. See Supplementary material.}

The next lemma shows that, if player $i+1$ wants to set $\alpha=\sigma^{i}-\omega_{i}$, one of the cheapest ways to buy $q-\left(\sigma^{i}-\omega_{i}\right)$ votes from $P_{i+1}$ involves buying player $i$ 's votes (equation (2)). Also, the benefit per vote of setting $\alpha=\sigma^{i}-\omega_{i}$ is as least as high as that of excluding player $i$ for $d_{i} \leq d_{i}^{*}$, with equality for $d_{i}=d_{i}^{*}$ (inequality (3)). This inequality plays a fundamental role in showing that $d_{i}^{*}$ is optimal: if the inequality would not hold, $i+1$ could obtain a higher benefit per vote by excluding $i$; if a strict inequality was possible for $d_{i}=d_{i}^{*}$, player $i$ could increase its demand without being excluded by $i+1$.

Lemma 7 Suppose we are in $\mathbb{B}(d, i)$. If $\sigma^{i}>\omega_{i}$ for some $\sigma^{i} \in \Sigma^{i}$ and player $i$ demands $d_{i} \leq d_{i}^{*}$, then

$$
b\left(i+1, \sigma^{i}-\omega_{i}\right)=b\left(i, \sigma^{i}\right)-d_{i}
$$

and, given any $\tau^{i} \in T^{i}$,

$$
\frac{b\left(i+1, \sigma^{i}-\omega_{i}\right)}{\sigma^{i}-\omega_{i}} \geq\left\{\begin{array}{cl}
\frac{b\left(i, \sigma^{i}\right)}{\sigma^{i}} & \text { if } \sigma^{i} \leq \delta^{i+1} \\
\frac{b\left(i, \tau^{i}\right)}{\tau^{i}} & \text { if } \sigma^{i}>\delta^{i+1} \text { and } b\left(i, \tau^{i}\right) \geq 0 \\
0 & \text { if } \sigma^{i}>\delta^{i+1} \text { and } b\left(i, \tau^{i}\right)<0 .
\end{array}\right\}
$$

Furthermore, the inequality in (3) is strict iff $d_{i}<d_{i}^{*}$.

Sketch of the proof. (See Supplementary material for details). Equation (2) is proven using lemma 6 . Suppose player $i+1$ sets $\alpha=\sigma^{i}-\omega_{i}$. If $\sigma^{i}-\omega_{i}<\gamma_{0}^{i}, i+1$ cannot form a winning coalition without $i$ 's votes and (2) follows regardless of $d_{i}$. Otherwise, we need to show that the maximum benefit from including player $i\left(b\left(i, \sigma^{i}\right)-d_{i}\right)$ is at least as high as the maximum benefit from excluding player $i\left(b\left(i, \sigma^{i}-\omega_{i}\right)\right)$. Because $\sigma^{i}-$ 
$\omega_{i} \leq \delta^{i+1}$, it is the case that $\frac{b\left(i, \sigma^{i}-\omega_{i}\right)}{\sigma^{i}-\omega_{i}} \leq \frac{b\left(i, \tau^{i}\right)}{\tau^{i}}$ (by definition of $\tau^{i}$ ) and $d_{i} \leq d_{i}^{*} \leq b\left(i, \sigma^{i}\right)-\frac{\sigma^{i}-\omega_{i}}{\tau^{i}} b\left(i, \tau^{i}\right)$ (because of the way $d_{i}^{*}$ is defined in (1)). Combining these two inequalities leads to $b\left(i, \sigma^{i}-\omega_{i}\right) \leq b\left(i, \sigma^{i}\right)-d_{i}$. Once (2) is established, (3) follows from $\frac{b\left(i+1, \sigma^{i}-\omega_{i}\right)}{\sigma^{i}-\omega_{i}}=\frac{b\left(i, \sigma^{i}\right)-d_{i}}{\sigma^{i}-\omega_{i}}$ and $d_{i} \leq d_{i}^{*}$.

The next lemma shows that if $d_{i}=d_{i}^{*}$, one of the values of $\alpha$ that maximize benefit per vote for $i+1$ is $\sigma^{i}-\omega_{i}$. There may be candidate coalitions for $i+1$ that do not involve $i$ (associated with $\sigma^{i}-\omega_{i}$ or with another $\sigma^{i+1} \in \Sigma^{i+1}$ ) but (2) implies that at least one coalition does.

Lemma 8 Suppose we are in $\mathbb{B}(d, i+1)$ and $\sigma^{i}>\omega_{i}$ for some $\sigma^{i} \in \Sigma^{i}$. If $d_{i}=d_{i}^{*}$, then $\sigma^{i}-\omega_{i} \in \Sigma^{i+1}$.

Sketch of the proof. (See Supplementary material for details). We need to prove that $\frac{b(i+1, \alpha)}{\alpha} \leq \frac{b\left(i+1, \sigma^{i}-\omega_{i}\right)}{\sigma^{i}-\omega_{i}}$ for all $\gamma^{i+1} \leq \alpha \leq \delta^{i+1}$. Because of lemma 6 , there are two possibilities for $b(i+1, \alpha)$. If $b(i+1, \alpha)=b(i, \alpha)$, the maximum benefit associated to $\alpha$ is achieved by excluding player $i$, and we know from lemma 7 that $\sigma^{i}-\omega_{i}$ leads to a higher benefit per vote than any alternative that excludes player $i$. If $b(i+1, \alpha)=b\left(i, \alpha+\omega_{i}\right)-d_{i}$, we can use inequality (3) to prove the result. There are several subcases to consider, depending on whether $\sigma^{i} \leq \delta^{i+1}, b\left(i, \tau^{i}\right) \geq 0$ and $\alpha+\omega_{i} \leq \delta^{i+1}$. For the case $\sigma^{i}>\delta^{i+1}$ and $\alpha+\omega_{i}>\delta^{i+1}$ the proof exploits the fact that, because of corollary $3, b\left(i, \alpha+\omega_{i}\right)=b\left(i, \sigma^{i}\right)$.

The next lemma makes a connection between the coalitions that maximize benefit per vote for player $i$ and the ones that do so for player $i+1$ when player $i$ sets $d_{i}=d_{i}^{*}$. If $i$ finds that buying the votes of coalition $S$ is optimal, $i+1$ will find that buying the votes of $S \cup\{i\}$ is optimal. Conversely, if $i+1$ finds that buying the votes of a coalition $S \ni i$ is optimal, $i$ also finds that buying the votes of $S \backslash\{i\}$ is optimal. There may be other coalitions that are optimal for $i+1$ and do not contain $i$ or the reverse (the latter happens if $\omega_{i} \in \Sigma^{i}$ ) but if we restrict attention to coalitions involving $i$ and $i+1$, exactly the same coalitions are optimal for $i$ and $i+1$. 
Lemma 9 Suppose we are in $\mathbb{B}(d, i+1), \sigma^{i}>\omega_{i}$ for some $\sigma^{i} \in \Sigma^{i}$ and $d_{i}=d_{i}^{*}$.

a) If $S \in \underset{T \subset P_{i}: \omega_{T} \geq q-\sigma^{i}}{\arg \max }\left(1-d_{T}\right)$ then $S \cup\{i\} \in \underset{T \subset P_{i+1}: \omega_{T} \geq q-\left(\sigma^{i}-\omega_{i}\right)}{\arg \max }\left(1-d_{T}\right)$ and $\sigma^{i}-\omega_{i} \in \Sigma^{i+1}$.

b) Given $\sigma^{i+1} \in \Sigma^{i+1}$ and $S \in \underset{T \subset P_{i+1}: \omega_{T} \geq q-\sigma^{i+1}}{\arg \max }\left(1-d_{T}\right), i \in S$ implies $S \cap P_{i} \in \underset{T \subset P_{i}: \omega_{T} \geq q-\sigma^{i}}{\arg \max }\left(1-d_{T}\right)$ for some $\sigma^{i} \in \Sigma^{i}$.

Sketch of the proof. (See Supplementary material for details). Part a) follows immediately from the previous two lemmas: it is optimal for $i+1$ to buy $\sigma^{i}-\omega_{i}$ votes (lemma 8) and to involve player $i$ (equation (2)). It remains to show that $i+1$ can buy the votes of $S \cup\{i\}$ to obtain this benefit, i.e. that $b\left(i+1, \sigma^{i}-\omega_{i}\right)=1-d_{S \cup\{i\}}$, which follows directly from (2) and $b\left(i, \sigma^{i}\right)=1-d_{S}$. For part b) we need to show that $b\left(i, \sigma^{i}\right)=$ $1-d_{S \cap P_{i}}$ for some $\sigma^{i} \in \Sigma^{i}$. Because $i \in S$, it follows from lemma 6 that $b\left(i+1, \sigma^{i+1}\right)=b\left(i, \sigma^{i+1}+\omega_{i}\right)-d_{i}$. Re-arranging this expression leads to $b\left(i, \sigma^{i+1}+\omega_{i}\right)=b\left(i+1, \sigma^{i+1}\right)+d_{i}=1-d_{S}-d_{i}=1-d_{S \cap P_{i}}$. Showing $\sigma^{i+1}+\omega_{i} \in \Sigma^{i}$ would complete the proof but this statement is not true in all subcases (if $b\left(i, \sigma^{i+1}\right)=0, b\left(i, \sigma^{i}\right)>0$ and $\omega_{i}>1, \delta^{i+1} \in \Sigma^{i+1}$ but $\left.\delta^{i+1}+\omega_{i} \notin \Sigma^{i}\right)$. The proof exploits the fact that we know the value of $\frac{b\left(i+1, \sigma^{i}-\omega_{i}\right)}{\sigma^{i}-\omega_{i}}$ from equation (3) and that $\sigma^{i}-\omega_{i} \in \Sigma^{i+1}$, so that (3) is also the value of $\frac{b\left(i+1, \sigma^{i+1}\right)}{\sigma^{i+1}}$. For the subcase $\sigma^{i} \leq \delta^{i+1}$, this knowledge can be used to show that $\sigma^{i+1}+\omega_{i} \in \Sigma^{i}$. For the subcase $\sigma^{i}>\delta^{i+1}$, it can be used to prove by contradiction that $b\left(i, \sigma^{i}\right)=1-d_{S \cap P_{i}}$ for some $\sigma^{i} \in \Sigma^{i}$.

The next lemma shows that a player that demands more than $d_{i}^{*}$ will be excluded from the next mover's planned coalition.

Lemma 10 Assume we are in $\mathbb{B}(d, i+1)$ and $d_{i}>d_{i}^{*}$.

a) If $b\left(i, \tau^{i}\right) \geq 0$ for some/all $\tau^{i} \in T^{i}$, then

$i \notin S$ for all $S \in \underset{T \subset P_{i+1}: \omega_{T} \geq q-\sigma^{i+1}}{\arg \max }\left(1-d_{T}\right)$ and all $\sigma^{i+1} \in \Sigma^{i+1}$.

b) If $b\left(i, \tau^{i}\right)<0$ for some/all $\tau^{i} \in T^{i}$, then every player obtains zero in any SPE of this subgame. 
Sketch of the proof. (See Supplementary material for details) a) If $d_{i}=d_{i}^{*}$, it follows from lemmas 7 and 8 that best option that involves buying the votes of player $i$ leads to the same benefit per vote as the best option that does not involve buying the votes of player $i$. If $d_{i}>d_{i}^{*}$, the best option that does not involve $i$ is unaffected whereas all options that involve player $i$ now have a lower benefit per vote.

b) For $b\left(i, \tau^{i}\right)<0$ the best alternative option is not to buy any votes at all, since buying votes would lead to a negative benefit. In this case $d_{i}^{*}=b\left(i, \sigma^{i}\right)$. Given that $b\left(i, \sigma^{i}\right) \geq 0, b\left(i, \tau^{i}\right)<0$ can only happen if $\sigma^{i}>\delta^{i+1}$. Then lemma 6 implies $b\left(i, \sigma^{i}\right)=b\left(i, \delta^{i}\right)$, that is, by setting $d_{i}=d_{i}^{*}$ player $i$ claims the entire available benefit. If $i$ sets $d_{i}>d_{i}^{*}$, all winning coalitions have a negative benefit and no coalition can be formed.

We are now in a position to use induction and show that the strategies we constructed constitute an SPE.

Proposition 2 The described strategies constitute an SPE of the demand bargaining game.

Sketch of the proof. (See Supplementary material for details) The proof shows by induction that it is optimal to form a coalition that maximizes benefit per vote and that players who demand $d_{i}^{*}$ will be included in the final coalition whereas players who demand more will be excluded. Lemmas 9 and 10 together with the tie-breaking rule provide the main basis for induction by showing that $i$ will be included by $i+1$ (and, by induction and the tie-breaking rule, by all subsequent movers) if it sets $d_{i}=d_{i}^{*}$ and excluded if it sets $d_{i}>d_{i}^{*}$.

\subsection{Showing uniqueness of SPE payoffs}

The next proposition shows uniqueness of equilibrium payoffs by backwards induction. Essentially we show that players must choose a coalition that 
maximizes the benefit per vote, and that ties must be solved in a certain way.

The uniqueness proof is based on the lemmas introduced so far plus a very simple additional lemma. Lemma 11 shows that, if $d_{i}<d_{i}^{*}$, all candidate coalitions for player $i+1$ involve player $i$. This result helps us prove that player $i$ can obtain $d_{i}$ by setting $d_{i}<d_{i}^{*}$ in any SPE (one would have to use induction to prove that $i+1$ can achieve the coalition it has in mind); hence $i+1$ must solve ties in $i$ 's favor when $d_{i}=d_{i}^{*}$ or $i$ would not have a best response.

Lemma 11 Suppose we are in $\mathbb{B}(d, i+1)$ and $\sigma^{i}>\omega_{i}$ for some $\sigma^{i} \in \Sigma^{i}$. If $d_{i}<d_{i}^{*}$, then for all $\sigma^{i+1} \in \Sigma^{i+1}$ and all $S \in \underset{T \subset P_{i+1}: \omega_{T} \geq q-\sigma^{i+1}}{\arg \max }\left(1-d_{T}\right)$ it holds that $i \in S$.

Proof. Let $\sigma^{i+1} \in \Sigma^{i+1}$. Suppose by contradiction that there exists a coalition $S \in \underset{T \subset P_{i+1}: \omega_{T} \geq q-\sigma^{i+1}}{\arg \max }\left(1-d_{T}\right)$ such that $i \notin S$. Then, $b\left(i+1, \sigma^{i+1}\right)=$ $b\left(i, \sigma^{i+1}\right)$ and

$$
\frac{b\left(i+1, \sigma^{i+1}\right)}{\sigma^{i+1}}=\frac{b\left(i, \sigma^{i+1}\right)}{\sigma^{i+1}} \leq \frac{b\left(i, \tau^{i}\right)}{\tau^{i}} \stackrel{(\text { Lemma }}{<} \frac{b\left(i+1, \sigma^{i}-\omega_{i}\right)}{\sigma^{i}-\omega_{i}} .
$$

which contradicts $\sigma^{i+1} \in \Sigma^{i+1}$.

Proposition 3 Assume we are in a SPE in $\mathbb{B}(d, i)$. If $b\left(i, \sigma^{i}\right) \geq 0$ for some/all $\sigma^{i} \in \Sigma^{i}$, player $i$ 's payoff is $d_{i}^{*}$ as defined in (1); otherwise player i's payoff is zero.

Proof. See Supplementary material.

Equilibrium strategies are not unique for some subgames. In subgames $\mathbb{B}(d, i)$ where no coalition can be formed (i.e., $\left.b\left(i, \sigma^{i}\right)<0\right)$, any demand vector is part of a SPE and equilibrium payoffs are always 0 for all players. Multiplicity may also arise in subgames where $b\left(i, \sigma^{i}\right)=0$, as the following example illustrates. 
Example 2 Consider the game $[5 ; 3,2,2,1,1]$ and suppose $d_{1}=d_{2}=1$. Equilibrium strategies at $\mathbb{B}(d, 3)$ are not unique, but equilibrium payoffs are.

At $\mathbb{B}(d, 3)$ we have $d_{3}^{*}=0$ and $\Sigma^{3}=\{2,3\}$. If we look at this subgame in isolation, several equilibrium outcomes are possible: coalition $\{1,3\}$ (associated to $\sigma^{3}=2$ ), coalition $\{2,3,4\}$ or $\{2,3,5\}$ (associated to $\sigma^{3}=3$ ), a coalition like $\{2,3,4,5\}$ (not a minimal winning coalition), coalition $\{1,4,5\}$ (which does not include player 3), or even no winning coalition at all. Intuitively, since the players in $\{3,4,5\}$ cannot get a positive payoff, they are indifferent between all these situations. However, players that have moved before are not indifferent. If we take into account that the strategies must be part of an equilibrium for all subgames, some of the equilibrium strategies at $\mathbb{B}(d, 3)$ are not equilibrium strategies for $\mathbb{B}(d, 2)$ and are discarded. A coalition containing player 2 must be formed in order for player 2 to have a best response at $\mathbb{B}(d, 2)$. Some multiplicity remains: after player 2 sets $d_{2}=1$, there are three possible equilibrium coalitions: $\{2,3,4\},\{2,3,5\}$ and $\{2,3,4,5\}$. Payoffs are identical in all three cases.

\section{Concluding remarks and discussion}

We have presented a demand bargaining model that makes sharp predictions regarding coalition formation and payoff division. The model can be extended to any finite horizon, and its predictions are independent of the discount factors and the risk attitudes of the players.

The result rests on two crucial assumptions: the order of moves ${ }^{14}$ and the game being well behaved (i.e., constant sum and homogeneous).

If we consider an arbitrary order of moves, it may be possible for one player to get everything (see Montero and Vidal-Puga, [22]). Multiple SPE are also possible. If we take the game $[3 ; 2,1,1,1]$ and order the players

\footnotetext{
${ }^{14}$ In a different setup with three players and partial agreements, Serrano [31] also finds that moving by decreasing power leads to the desired payoff vector (the nucleolus).
} 
in such a way that the player with 2 votes is in the third place, no set of consecutive movers of the form $\{1, \ldots, i\}$ is a minimal winning coalition. If players move in this order, the first mover cannot get a positive payoff for any demand, and this leads to a continuum of subgame perfect equilibria.

When players move by decreasing weight, the coalition that forms is the minimal winning coalition with the smallest number of players. One may ask whether proportional payoffs can be achieved for an arbitrary minimal winning coalition by choosing the order of moves appropriately. This is sometimes but not always possible. ${ }^{15}$ In the game $[4 ; 2,2,1,1,1]$, a coalition of type [211] forms with a proportional division if the order of moves is of type [21121]). In the apex game $[3 ; 2,1,1,1]$, there is no order of moves for which coalition $\{2,3,4\}$ forms with a proportional payoff division. This coalition can only form if the large player moves last, but then the first mover is able to get everything. ${ }^{16}$ The intuitive reason why proportionality fails is that, after the first mover makes a larger than proportional demand, we cannot find a minimal winning coalition moving consecutively and immediately after the first mover, hence coordinating a reaction is difficult and players moving after the first mover may end up sabotaging each other's plans.

If the game is not constant-sum and homogeneous, proportionality may break down. In some cases, this is due to the presence of a player that can be "held hostage" by others, as pointed by Morelli [23].

Example 3 There are four players, with 3,2,2 and 1 votes respectively. The quota is 5 . If the players play a demand bargaining game in decreasing order, any subgame perfect equilibrium results in coalition $\{1,2\}$ with $d_{1}=\frac{1}{2}$

\footnotetext{
${ }^{15}$ For the Baron-Ferejohn model, Kalandrakis [17] shows that any payoff vector can be obtained by manipulating the proposer probabilities. This result is not replicated for fixed order demand bargaining, even though many payoff vectors are feasible due to the multiplicity of SPE for some orders.

${ }^{16}$ Proportionality for all coalitions in apex games can be restored if the order of moves is not determined in advance, but the next mover is randomly selected (Fréchette et al, [15]). How far this result extends beyond apex games is an open question.
} 
and $d_{2}=\frac{1}{2}$.

Player 4 is helpless because there is only one minimal winning coalition it can form. Knowing this, player 3 will either form a coalition with 1 and get $1-d_{1}$, or set $d_{3}=1-d_{2}$. Player 2 can then form a coalition with 1 (obtaining $1-d_{1}$ ) or set $d_{2}=d_{1}$ and induce coalition $\{2,3,4\}$. Anticipating this, player 1 sets $d_{1}=\frac{1}{2} \cdot{ }^{17}$ The game $[5 ; 3,2,2,1]$ has many homogeneous representations, but in none of them do players 1 and 2 have the same number of votes. In terms of our proof, player 3 is not choosing a coalition with the maximum benefit per vote, but is biased towards coalitions that include player 4 . This sort of situation could never happen in a constantsum game, because there $\omega_{i} \leq \delta^{i+1}$ for all $i$, and 4 and its followers would be able to form an alternative coalition with player 1 . Note also that lemma 2 does not hold for this game.

Proportionality can break down even if no player can be held hostage by others, as the following example illustrates.

Example 4 Consider the game $[7 ; 4,3,2,2,1,1]$. If the players play a demand bargaining game in decreasing order, any subgame perfect equilibrium results in coalition $\{1,2\}$ with $d_{1}=d_{2}=\frac{1}{2}$.

The game above is constant-sum but not homogeneous. None of the players can be held hostage by others: given any two players, each of them can form a minimal winning coalition that does not include the other. Moreover, coalition $\{1,2\}$ has exactly 7 votes. Nevertheless, proportionality fails because $\{1,3,4\}$ and $\{2,3,4\}$ are both minimal winning coalitions. From the point of view of players 3 and 4 , players 1 and 2 are equally valuable even though they have a different number of votes. If the turn reaches player

\footnotetext{
${ }^{17}$ The prediction that 4 should get 0 even if coalition $\{2,3,4\}$ forms is well grounded in cooperative game theory. The bargaining set (see Maschler, [19]) for coalition $\{2,3,4\}$, the aspiration solution concepts of Bennett [4] and the inferior player axiom of Napel and Widgrén [25] all agree in making this prediction.
} 
3 , which of the two coalitions forms will depend on whether $d_{1}$ is higher or lower than $d_{2}$. Anticipating this, player 2 has two options: it can form a coalition with 1 and get $1-d_{1}$, or set $d_{2}=d_{1}$ and induce coalition $\{2,3,4\}$. Player 2 will form a coalition if $1-d_{1} \geq d_{1}$, or $d_{1} \leq \frac{1}{2}$. Knowing this, player 1 sets $d_{1}=\frac{1}{2}$. Note that player 4 does not always choose the coalition that maximizes benefit per vote; this criterion is not reliable for nonhomogeneous games as it may lead to $\sigma^{4}<\omega_{4}$. Also, lemma 2 does not hold for player 4 . 


\section{References}

[1] Austen-Smith, D. and Banks, J. (1988) Elections, Coalitions and Legislative Outcomes. American Political Science Review 82, 405-422.

[2] Banks, J.S. and Duggan, J. (2000) A Bargaining Model of Collective Choice. American Political Science Review 94, 73-88.

[3] Baron, D. and Ferejohn, J. (1989) Bargaining in Legislatures. American Political Science Review 87, 34-47.

[4] Bennett, E. (1983). The Aspiration Approach to Predicting Coalition Formation and Payoff Distribution in Sidepayment Games. International Journal of Game Theory 12, 1-28.

[5] Bennett, E. and van Damme, E. (1991) Demand Commitment Bargaining: The Case of Apex Games. Game equilibrium models III: Strategic bargaining. Ed. by R. Selten. Berlin. Springer-Verlag, 118-140.

[6] Binmore, K.G. (1985) Bargaining and Coalitions. In: Roth (ed) Game theoretic models of bargaining, Cambridge: Cambridge University Press.

[7] Binmore, K. (1987). Perfect Equilibria in Bargaining Models in The Economics of Bargaining, ed by K. Binmore and P. Dasgupta.

[8] Bloch, F. and Rottier, S. (2002) Agenda Control in Coalition Formation. Social Choice and Welfare 19, 769-788.

[9] Cardona-Coll, D. and Mancera, F. M. (2000). Demand Bargaining in Legislatures. Economic Theory 16, 163-180.

[10] Chatterjee, K., Dutta, B., Ray, D. and Sengupta, K. (1993). A Noncooperative Theory of Coalitional Bargaining. Review of Economic Studies 60, 463-77. 
[11] Cross, J. (1967). Some Theoretical Characteristics of Economic and Political Coalitions. Journal of Conflict Resolution 11, 184-195.

[12] Dasgupta, A. and Chiu, Y.S. (1998) On Implementation via Demand Commitment Games. International Journal of Game Theory 27, 161189.

[13] Diermeier, D., Eraslan, H. and Merlo, A. (2003). A Structural Model of Government Formation. Econometrica 71, 27-70.

[14] Diermeier, D. and Merlo, A. (2004) An Empirical Investigation of Coalitional Bargaining Procedures. Journal of Public Economics 88, 783-797.

[15] Fréchette, G., Kagel, J. and Morelli, M. (2005) Behavioral Identification in Coalitional Bargaining: An Experimental Analysis of Demand Bargaining and Alternating Offers. Econometrica 73, 1893-1937.

[16] Gamson, W.A. (1961) A Theory of Coalition Formation. American Sociological Review 26, 373-382.

[17] Kalandrakis, T. (2006). Proposal Rights and Political Power. American Journal of Political Science 50, 441-448.

[18] Laver, M. and Schofield, N. (1990) Multiparty Government: the Politics of Coalition in Europe. Oxford: Oxford University Press.

[19] Maschler, M. (1992). The Bargaining Set, Kernel, and Nucleolus, in Handbook of Game Theory with Economic Applications, volume 1, ed. by R.J. Aumann and S. Hart. North-Holland, 1992, 591-667.

[20] McKelvey, R. D., Ordeshook, P. C. and Winer, M. D. (1978) The Competitive Solution for N-Person Games without Transferable Utility, with an Application to Committee Games. American Political Science Review 72, 599-615. 
[21] Montero, M. (2006) Noncooperative Foundations of the Nucleolus in Majority Games. Games and Economic Behavior 54, 380-397.

[22] Montero, M. and Vidal-Puga, J. J. (2007) Demand Commitment in Legistative Bargaining, American Political Science Review 101 (4) 847850.

[23] Morelli, M. (1999) Demand Competition and Policy Compromise in Legislative Bargaining. American Political Science Review 93, 809-820.

[24] Morelli, M. and Montero, M. (2003) The Demand Bargaining Set: General Characterization and Application to Majority Games, Games and Economic Behavior 42, 137-155.

[25] Napel, S., Widgrén, M. (2001). Inferior Players in Simple Games. International Journal of Game Theory 30, 209-220.

[26] Norman, P. (2002). Legislative Bargaining and Coalition Formation, Journal of Economic Theory 102, 322-353.

[27] Ostmann, A. (1987) On the Minimal Representation of Homogeneous Games. International Journal of Game Theory 16, 69-81.

[28] Peleg, B. (1968) On Weights of Constant-Sum Majority Games. SIAM Journal of Applied Mathematics 16, 527-532.

[29] Rubinstein, A. (1982) Perfect Equilibrium in a Bargaining Model. Econometrica 50 , 97-108.

[30] Selten, R. (1992) A Demand Commitment Model of Coalition Bargaining. Rational interaction, Essays in honor of John C. Harsanyi. Ed. by R. Selten.

[31] Serrano, R. (1993). Noncooperative Implementation of the Nucleolus: the 3-Player Case. International Journal of Game Theory 22, 345-357. 
[32] Vidal-Puga, J.J. (2004) Bargaining with Commitments. International Journal of Game Theory 33, 129-144.

[33] Von Neumann, J. and Morgenstern, O. (1944). Theory of Games and Economic Behavior, Princeton N.J., Princeton University Press.

[34] Winter, E. (1994) The Demand Commitment Bargaining and Snowballing Cooperation. Economic Theory 4, 255-273. 